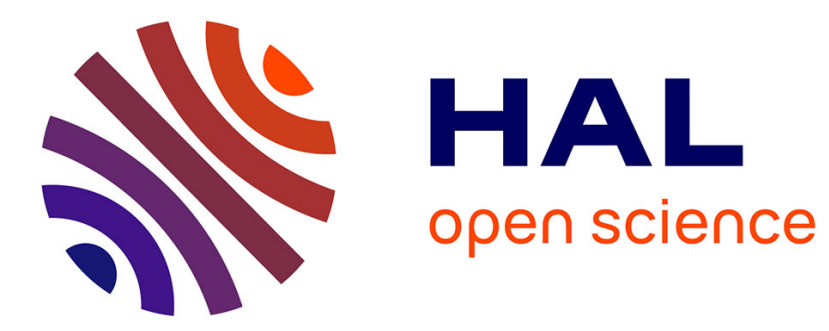

\title{
Multi-UAVs network communication study for distributed visual simultaneous localization and mapping
}

Nesrine Mahdoui, Enrico Natalizio, Vincent Frémont

\section{To cite this version:}

Nesrine Mahdoui, Enrico Natalizio, Vincent Frémont. Multi-UAVs network communication study for distributed visual simultaneous localization and mapping. International Workshop on Wireless Sensor, Actuator and Robot Networks (ICNC 2016), Feb 2016, Kauai, United States. pp.1-5, 10.1109/ICCNC.2016.7440564 . hal-01399793

\section{HAL Id: hal-01399793 https://hal.science/hal-01399793}

Submitted on 20 Nov 2016

HAL is a multi-disciplinary open access archive for the deposit and dissemination of scientific research documents, whether they are published or not. The documents may come from teaching and research institutions in France or abroad, or from public or private research centers.
L'archive ouverte pluridisciplinaire HAL, est destinée au dépôt et à la diffusion de documents scientifiques de niveau recherche, publiés ou non, émanant des établissements d'enseignement et de recherche français ou étrangers, des laboratoires publics ou privés. 


\title{
Multi-UAVs Network Communication Study for Distributed Visual Simultaneous Localization and Mapping
}

\author{
Nesrine Mahdoui, Enrico Natalizio and Vincent Fremont \\ Université de Technologie de Compiègne (UTC) \\ CNRS Heudiasyc UMR 7253 \\ Compiègne, France \\ Email: firstname.lastname@hds.utc.fr
}

\begin{abstract}
In this paper, the problem of multi-UAVs (Unmanned Arial Vehicles) Visual Simultaneous Localization and Mapping (SLAM) is considered by using a new framework for pose and map estimation using monocular vision and reduced communications capabilities. The problem of localization and mapping is solved by fusing monocular visual data with odometry measurements through Graph SLAM formulation. Using each robot's map data representation, a proposal is made for a good and robust communication between UAVs to perform efficient data exchange while keeping SLAM performances. A mesh network is chosen to import solutions to wireless networking. Finally, some validation experiments are performed in an Ad Hoc Network and a Wireless Mesh Network using Better Approach To Mobile Ad Hoc Network (BATMAN) protocol.
\end{abstract}

\section{INTRODUCTION}

In the recent years, important progress have been done in the field of cooperative SLAM for multi-UAVs systems. Teams of UAVs are used to realize several missions like security, surveillance, search and rescue and autonomous navigation in unknown environments. In each mission, the common feature is to guarantee an autonomous and coordinated exploration. Using collaborative fleet of UAVs instead of a single UAV offers several advantages to achieve mission's goals. We can mention, at first, an increasing scalability of the operations, which means that the multi-UAVs system helps covering a larger area. Also, a group of UAVs can limit the risks of mission failure. In fact, if a member of the group does not perform correctly its task, it can rely on the others to replace it. In another way, the mission execution time will be much lower with a multi-UAVs system than with a single UAV, as the UAVs will be able to rely on each other to speed up the process. That is why cooperative groups can remarkably increase the task's performances. Nevertheless, although the important advances in the deployment of a team of UAVs, such operations still present some difficulties. The main problem is to implement a cooperative, distributed and vision-based algorithm to coordinate the UAVs to reach the missions goal. Without a global positionning system, the first challenge is to perform a robust Visual Localization and Mapping especially in the monocular case where the scale ambiguity appears. Indeed, using only Inertial Measurement Unit (IMU) information, the position may rapidly drift. Therefore, the combination of IMU and camera seems to be adequate to provide a more accurate map of UAV's environment with a consistent scale. In this paper, we propose to use the Incremental Smoothing and Mapping (iSAM2) algorithm to build the map [1]. As a concept, we consider the global map result as a submap merging of the near covered environment by each UAV. In a cooperative work, the task does not stop upon the creation of a reliable submap, but it extends to exchange information in a safe way between members of the team. We need to ensure an efficient networking support, related to mission conditions, which can overcome communication losses, node failures, changes in network topology. In the literature, several works on Visual SLAM [2], [3] and on networking scheme for UAV communication [4], [5], [6], [7] have been presented, but it is rare to find studies on both topics together [8]. Therefore, our main purpose is to construct a distributed, and cooperative multi-UAVs visual SLAM system focusing on both communications, localization and mapping process. The proposed SLAM framework allows us to create a submap for each UAV and to share the collected data between UAVs through a mesh network. It is also important to ensure an on board computation capability to save time and to speed up the mission. So our goal is to study a reliable network configuration respecting the SLAM constraints and to study both topics in parallel.

The paper contributions are the following:

- A Monocular Visual Graph SLAM approach is introduced: The iSAM2, a sparse non linear incremental algorithm, has shown his efficiency to solve SLAM problem with stereo camera.In this paper, we propose a new architecture, using an IMU and a monocular camera to build each UAV's submap.

- The use of mesh network mechanisms in UAVs communications taking into account the SLAM's requirements is also presented: Our contribution here, is to combine SLAM requirements along with communication schemes by using an appropriate network architecture.

- Broadcast data in testbeds: we compare, with testbeds, the use of a simple Ad Hoc network versus the use of a mesh network that uses Better Approach To Mobile Ad Hoc Network (BATMAN).

This paper is organized as follows: in Section 2, we present the related works. The system architecture follows in Section 3. The experimental results are then presented in Section 4 and the conclusion and future works are given in Section 5. 


\section{RELATED WORKS}

The SLAM is one of the most popular topic in the robotics community. To solve the problem of multi-UAV SLAM, some works proposed an approach enabled by pairwise relations between UAVs to fuse information and construct a distributed map [9].

In a similar approach to build a distributed SLAM architecture, an extending Smoothing And Mapping (SAM) approach is used. It is a graphical model approach that introduces the Constrained Factor Graph (CFG) [10]. To realize a distributed system, they improved the naive approach by building a Decentralized Data Fusion- SAM that satisfies the DDF requirements while keeping the benefits of the naive approach.

For single robot visual SLAM the Parallel Tracking and Mapping (PTAM) approach has shown promising results. It is based on a parallel framework as tracker and mapper to increase the responsiveness and the robustness of the whole system [11]. The tracker enables fast camera localization in real time, during this, the keyframe based mapper builds the global map. The PTAM, originally designed for augmented reality, was modified making it more suitable for UAV navigation [12]. It uses odometry measurements instead of motion model and fuses the visual and odometry measurements to deal with the lack of visual features and the lack of motion in the environment. The approach integrates also a loop closer mechanism compared to the originally PTAM method.

In a collaborative monocular SLAM study, the work combines an onboard camera and an IMU on each UAV allowing it to determine its own individual motion [2]. The image coordinates and descriptors like features of selected keyframes and relative pose estimates are streamed to a ground station called the Collaborative Structure From Motion. This CSFM creates the map for each single UAV and merge them whenever there is an overlap. Similarly, for visual SLAM problem, a software architecture concept was made for unknown environment and based on IMU and a pair of camera to create the UAV's map [13]. An on-board stereo-vision based mapping system was tested in an indoor environment and proved its effectiveness with less than $0.08 \%$ deviation from the ground truth.

Choosing only the SLAM algorithm is not sufficient enough to create a distributed, collaborative and visual fleet of UAV, but we need also to determine the appropriate way for communication inter-UAV. A large body of related work exists; among it, we can find the Optical Wireless Communication (OWC) [14]-[16]. But, the most common network used in multiUAVs system is the Ad Hoc network. This network present different architecture like MANET (Mobile Ah doc NETwork), VANET (Vehicular Ah doc NETwork) and the FANET (Flying NETworks). Between them FANET is chosen because of the high mobility degree, random mobility model, topology change and an important distance between nodes [17].

The network could be: centralized [2], [4], where data must go through the central node which makes the network vulnerable. It may be also decentralized [9], [13], where it is considered as a set of centralized networks. The distributed network, is considered as a solution for the problem of frailty of both previous networks due to links present over all nodes that makes a reliable communication [8], [18], [19]. The Ad Hoc network is an infrastructure less mode, also called mesh mode as we can confuse both designation. Some works use the $802.11 \mathrm{~s}$ mesh network to perform a centralized system architecture with a distributed communication and centralized manner in case of failure of the first one where communication is able to satisfy some specific QoS, reduce the traffic and increase the response time and robustness [8]. The 802.11s mesh network is also used to connect two UAV and the 802.11g between the UAV and the computer as mesh access point [4]. Moreover, we need to choose an adequate protocol that guarantee the required QoS. With the multi-hop 802.11n, experiments were performed in lab between BATMAN, OLSR and AODV routing protocols where BATMAN outperforms others and an in-flight experiment between BATMAN and geo-routing that was more efficient in the performance of transmission [7]. Also, we may build a wireless mesh network as wireless backbone within the areas of special interest [6]. The authors believes that integrating UAV swarms with wireless mesh networking provides a new solution of effective and interactive border surveillance.

\section{System ARCHITECTURE}

Most of the existing works study the communication aspect and the SLAM algorithms separately. In this paper, we try to optimize the perception and the communication part in a cooperative way. We formulate a system that satisfy the SLAM requirements while keeping the network advantages. Usually, practical solutions share images between UAV, however, network throughput is negatively affected by these solutions. Therefore, we decided to design a new framework to make possible the exchange of maps instead of images. The Fig. 1 illustrates the proposed architecture for the SLAM.

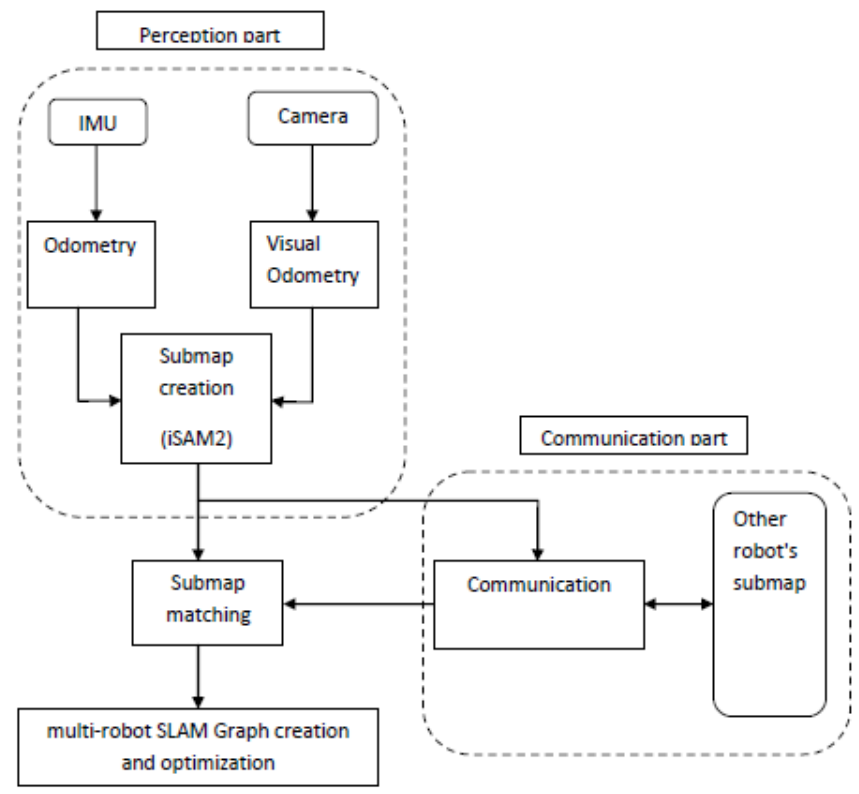

Figure 1. Proposed software architecture 
In this overview, we use both an IMU and a camera. The IMU allows us to collect the odometry measurements between two consecutive poses of the UAV but the resulting trajectory may rapidly deflect, so we make use of a monocular camera to provide a more stable trajectory result through visual odometry. Once one time step data are available, the iSAM2 algorithm is used to construct the robot's map. This map is considered, as a submap that is composed of the UAV's trajectory as well as landmarks. The submap is then exchanged between UAVs where each one sends its own map through the communication module. Every UAV manages a structure containing all the submaps georeferenced relatively to their owner in the fleet. This arrangement leads, for a single UAV, the possibility of merging these submaps to create a global map. The submap matching part is not treated in this work because in this research, we focus on Visual SLAM and communication aspects.

\section{A. Perception Process}

To perform the UAV's submap calculation, an IMU and a monocular camera are used as the main sensors. Indeed, the odometry measurements are used in place of a motion model to build the trajectory. The IMU outputs odometry between two consecutive poses of the UAV. The estimated poses being not reliable, the camera reinforces it through visual odometry. Both IMU data and visual odometry are merged in the submap calculation module with the iSAM2 approach to remove the overall scale factor ambiguity. The specificity of the iSAM2 approach is that when new measurements are available, it provides updated estimates during the UAV's activity.

In the proposed framework, the SLAM problem is represented as a factor graph. It is presented as a factorization of a function $\mathrm{f}(\theta)$ as $\mathrm{f}(\theta)=\prod_{i} f\left(\theta_{i}\right)$ with $\theta_{i}$ as variable nodes and the purpose is to find the variable that maximize $\theta^{*}=\operatorname{argmax}_{\theta} f(\theta)$. The factor graph, presented in Fig. 2 , is composed of unknown poses $\mathrm{X}=\left\{x_{i}\right\}_{i=1}^{n}$, landmarks $\mathrm{L}=\left\{l_{i}\right\}_{j=1}^{m}$, visual measurements $\mathrm{Z}=\left\{z_{i j}\right\}_{j=1, i=c s t e}^{m}$ and odometry measurements $\mathrm{B}=\left\{b_{i}\right\}_{i=1}^{n-1}$ with $\mathrm{b}_{i}$ the odometry between the pose $\mathrm{x}_{i}$ and $\mathrm{x}_{i+1}$. These variables are classified in variable nodes and factor nodes due to the bipartite aspect of the factor graph. The camera pose $x_{i}$ and the landmarks $1_{j}$ are presented as variable nodes in the graph while the factor nodes are: the prior densities $\mathrm{p}\left(\mathrm{x}_{0}\right)$ on the variable nodes, the motion models between two camera poses $\mathrm{p}\left(\mathrm{x}_{i+1} \mid x_{i}, b_{i}\right)$, given the odometry measurement $\mathrm{b}_{i}$ and the measurement likelihood models $\mathrm{p}\left(\mathrm{z}_{i j} \mid x_{i}, l_{j}\right)$ between the pose $\mathrm{x}_{i}$ and the landmark $\mathrm{l}_{j}$ given the visual measurement $\mathrm{z}_{i j}$.

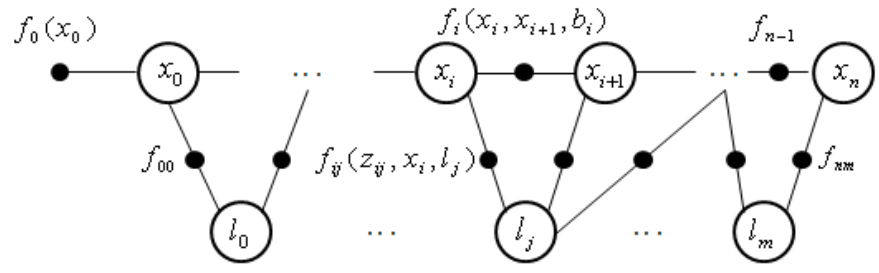

Figure 2. Factor graph in monocular SLAM where $\mathrm{x}_{i}$ and $1_{i}$ are the variable nodes and $\mathrm{f}_{0}$ : prior density factor, $\mathrm{f}_{i}$ : odometry factor between pose $\mathrm{x}_{i}$ and $\mathrm{x}_{i+1}$ and $\mathrm{f}_{i j}$ : the measurements likelihood model between a pose $\mathrm{x}_{i}$ and its landmark $1_{i}$.

The estimation problem is actually based on three graphical models: an explicit factor graph, an implicit chordal Bayes net and a Bayes tree. Therefore, the iSAM2 algorithm can obtain incrementally an estimate for unknown variables, like the UAV poses and the landmarks, given a set of non linear factors as odometry to finally construct the UAV's sub map.

This implementation considerably decreases the data size from exchanging image to exchanging maps in presence of limited communication bandwidth. In addition, our structure allows each UAV to construct its own graph and submap separately by adding the measurements only once. We can then avoid the double counting of data. The iSAM2's factor graph structure could also be used, in further work, to add other constraints such as loop closing, dynamic objects or mesh network requirements.

\section{B. Communication Block}

The communication module is a crucial module in the fleet of UAVs. It should be chosen following some requirements that are able to deal with the following challenges: the development of the software needed to efficiently monitor the UAVs and the protocol design to coordinate UAVs that move continuously [6]

Our network have to ensure that the information circulate properly among the nodes by choosing an adequate standard. Those standards refer to the two lowest layer in the Open Systems Interconnection (OSI) architecture. The network chosen shall overcome:

- Node failure: when the link is broken or defected, network must find a path to reach the node.

- Topology changes: nodes move in the environment and generate changes in the topology that the network have to deal with rapidly.

- Communication bandwidth: UAVs possess certain information to exchange with other and need some bandwidth that supports the data exchange.

To ensure a completeness between the visual SLAM and the communication, we choose a distributed and cooperative wireless network. Firstly, based on a comparison in table 1, we decide to opt for the infrastructure less mode instead of the infrastructure mode to create a fully distributed SLAM. 


\begin{tabular}{|c|c|c|}
\hline & $\begin{array}{l}\text { Infrastructure mode } \\
\text { (Access Point mode) }\end{array}$ & $\begin{array}{l}\text { Infrastructure less } \\
\text { (mesh mode) }\end{array}$ \\
\hline Advantages & $\begin{array}{l}\text { reliability of } \\
\text { communication, }\end{array}$ & $\begin{array}{l}\text { direct connection to } \\
\text { each other, easy to } \\
\text { set up, robust to node } \\
\text { failure, allow } \\
\text { expansion and } \\
\text { modification in } \\
\text { network topology }\end{array}$ \\
\hline Disadvantages & $\begin{array}{c}\text { expensive, } \\
\text { complicated } \\
\text { hardware, range } \\
\text { restriction }\end{array}$ & $\begin{array}{c}\text { redundancy in } \\
\text { network connection, } \\
\text { difficult maintenance }\end{array}$ \\
\hline Standard & 802.11 & $\begin{array}{c}802.11,802.15, \\
802.16\end{array}$ \\
\hline
\end{tabular}

INFRASTRUCTURE AND INFRASTRUCTURE LESS MODE

In the infrastructure less mode, we find the kind of network interesting for the following advantages:

- Improves the network reliability because the mesh network offers several paths so that information may reach destination even if one link is broken.

- Improves performances by balancing the traffic on the Access Point.

- Allows self scalability of the network by adapting quickly to changes in network topology.

- Enables rapid deployment with lower cost backhaul.

- Provides easily coverage in areas difficult to access.

- Saves battery life due to his lower power consumption.

The IEEE $802.11 \mathrm{n}$ presents more important performances compared to $802.11 \mathrm{~s}$ mesh network but its throughput decreases with the increased number of hops [20].

To make a compromise between hardware and software requirements, solutions involving mesh networks are the most interesting. Among them, the $802.11 \mathrm{~s}$ amendment, related to the MAC layer, is an Extended Service Set network that supports broadcast, multicast and unicast communication. It contains the Hybrid Wireless Mesh Protocol (HWMP) as default routing protocol. The HWMP is an hybrid routing protocol inspired by the AODV an on demand and reactive portion and the tree based protocol a proactive portion. Thereby, it contains the advantages of the reactive protocol as it prepares the routing table when nodes change their position and gives the most safe path and on the other hand, the advantages of the proactive protocol as the routing table is ready and we save time when needed. In addition to the default routing protocol HWMP in the 802.11s, mesh network may support other protocols like Optimized Link State Routing (OLSR), BATMAN, Wireless Distribution System (WDS), Open Shortest Path First (OSPF) and BABEL. BATMAN proved better performances than HWMP and OLSR [21], thus, we decided to make experimentations by using the HWMP and BATMAN.

\section{EXPERIMENTAL RESULTS}

\section{A. Visual SLAM Performances}

We implemented the SLAM algorithm with the Georgia Tech Smoothing And Mapping (GTSAM) toolbox ${ }^{1}$ using the factor graph implementation. The experimental code is written in Matlab and includes mex functions of the c++ library. We performed tests on a $2.50 \mathrm{GHz}$ i5 Linux machine. To validate the SLAM algorithm, we simulated the measurements with raw data from the Kitti vision dataset ${ }^{2}$. These data contain IMU measurements used for dead reckoning and images used for the visual features. For this, the libviso 2 library ${ }^{3}$ is used to compute the inter frame visual odometry. The iSAM2 then estimates and optimize poses and landmarks given these set of non linear factors as IMU and visual odometry mesurements.

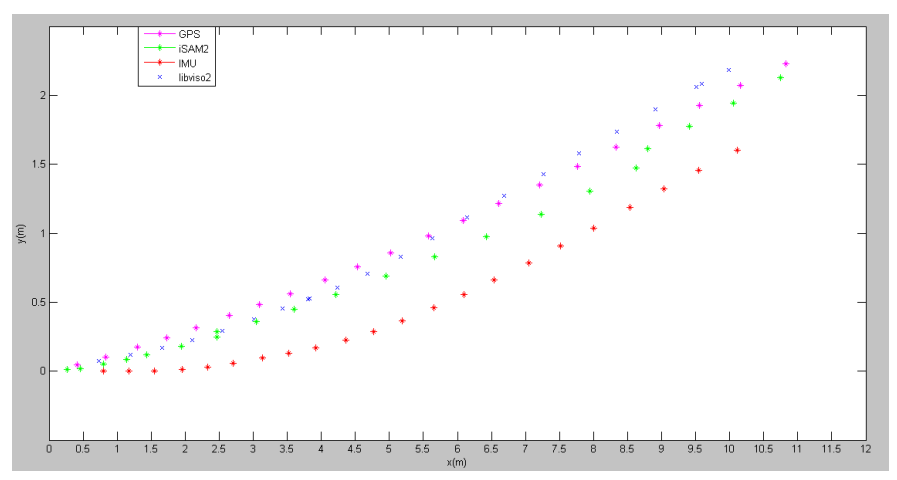

Figure 3. Trajectory results (2D projection) using IMU, libviso2, iSAM2 and the GPS trajectory.

Results in Fig. 3 shows that combining the IMU and the libviso 2 trajectory using the iSAM2 helps to improve the poses estimation, and to reduce the IMU drift compare to gound truth.

\section{B. Distributed SLAM for multi UAVs}

For the evaluation of the distributed SLAM system, we use heterogenous nodes in the network composed of three laptops : $2.40 \mathrm{GHz}$ dual core Linux machine, $2.27 \mathrm{GHz}$ i3 Linux machine, $2.50 \mathrm{GHz}$ i5 Linux machine and a Parrot AR DRONE 2.0 (see Fig. 4). We recover results data from the previous SLAM test and simulate the files broadcast between nodes in both Ad Hoc and mesh network to underline the benefit.

\footnotetext{
${ }^{1}$ https://collab.cc.gatech.edu/borg/gtsam?destination=node $\% 2$ F299

${ }^{2}$ http://www.cvlibs.net/datasets/kitti/raw_data.php

${ }^{3}$ http://www.cvlibs.net/software/libviso/
} 


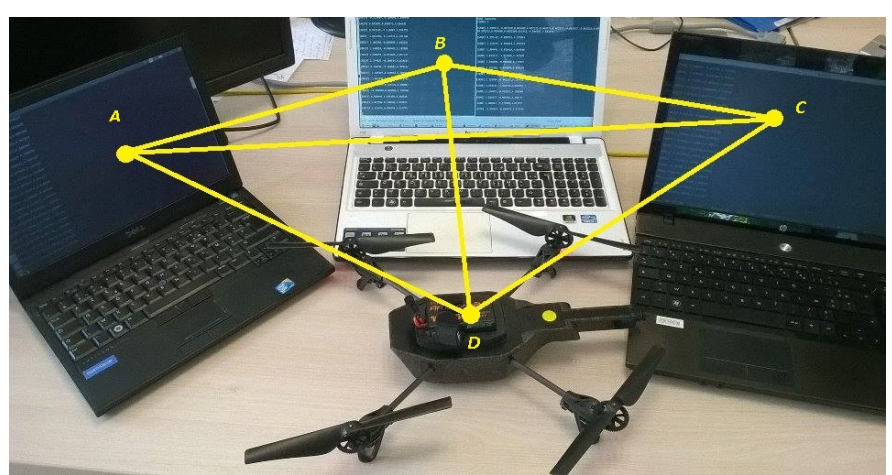

Figure 4. Mesh network illustration between laptops and drone.

The drone was controlled from the laptop by using cross compilation. First, we perform an Ad Hoc network between end points and broadcast the SLAM data file from node A to nodes $\mathrm{B}, \mathrm{C}$ and $\mathrm{D}$ in the network. Then, we broadcast, in the same conditions, from node $\mathrm{A}$ to $\mathrm{B}, \mathrm{C}$ and $\mathrm{D}$ with a BATMAN mesh network protocol.

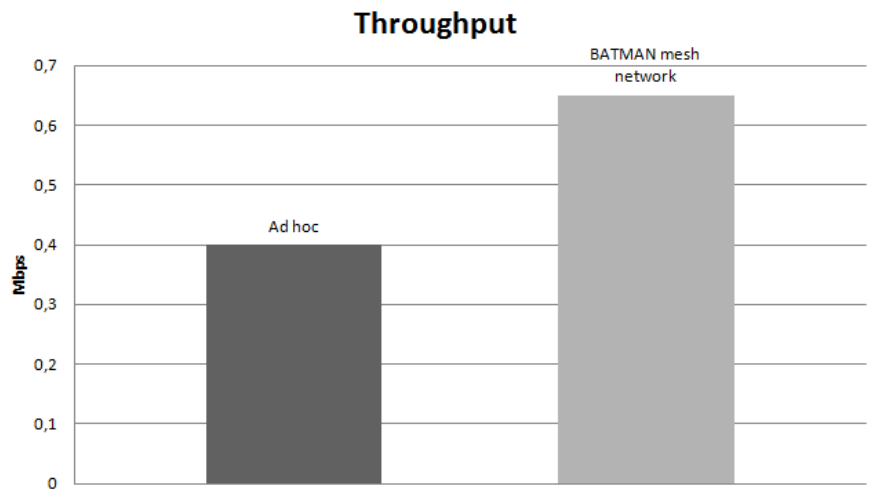

Figure 5. Broadcast testbed throughput result in Ad Hoc and BATMAN mesh network protocol.

The results in Fig. 5, shows that the throughput achieved an average of $0.4 \mathrm{Mbits} / \mathrm{s}$; whereas, the throughput evaluated in mesh network with BATMAN protocol, achieved an average of $0.65 \mathrm{Mbits} / \mathrm{s}$. The BATMAN mesh network protocol improves the throughput of the network.

\section{CONClusions AND Future WORKS}

This paper proposed a software architecture for cooperative and distributed visual SLAM. The proposed architecture improved the IMU trajectory and makes it closer to the ground truth by combining it with visual odometry within a Graph SLAM framework.

We implemente a testbed consisting of heterogeneous nodes: three laptops and one drone. We compared the performances of a simple Ad Hoc network versus a mesh network using the BATMAN protocol. The BATMAN mesh network protocol has a better performance in terms of throughput, which is about 1.5 times the throughput of the Ad Hoc network.

As future works, we plan to implement our approach on a UAV to evaluate the realtime performances. We also plan to add constraints to the iSAM2 factor graph as loop closure, dynamic features or mesh network topology. Another challenge that will be treated in future works, is to merge the submaps obtained by each UAV to build the global map of the environment.

\section{ACKNOWLEDGMENTS}

This work is supported by the Labex MS2T, funded by the French Government through the program "Investments for the future" managed by the National Agency for Research (Reference ANR-11-IDEX-0004-02).

\section{REFERENCES}

[1] Michael Kaess, Hordur Johannsson, Richard Roberts, Viorela Ila, John J Leonard, and Frank Dellaert. isam2: Incremental smoothing and mapping using the bayes tree. The International Journal of Robotics Research, 2012.

[2] C. Forster, S. Lynen, L. Kneip, and D. Scaramuzza. Collaborative monocular slam with multiple micro aerial vehicles. In Intelligent Robots and Systems (IROS), 2013 IEEE/RSJ International Conference. IEEE, 2013.

[3] Danping Zou and Ping Tan. Coslam: Collaborative visual slam in dynamic environments. Pattern Analysis and Machine Intelligence, IEEE Transactions on (Volume:35, Issue: 2 ), 2013.

[4] Simon Morgenthaler, Torsten Braun, Zhongliang Zhao, Thomas Staub, and Markus Anwander. Uavnet: A mobile wireless mesh network using unmanned aerial vehicles. Globecom Workshops (GC Wkshps),IEEE, 2012.

[5] Samira Hayat, Evsen Yanmaz, and Christian Bettstetter. Experimental analysis of multipoint-to-point uav communications with ieee $802.11 \mathrm{n}$ and 802.11ac. Technical report, Institute of Networked and Embedded Systems, Alpen-Adria-Universitšat Klagenfurt, Austria and Lakeside Labs GmbH, Klagenfurt, Austria, 2015.

[6] Ming Li, Kejie Lu, Hua Zhu, Min Chen, Shiwen Mao, and B. Prabhakaran. Robot swarm communication networks: Architectures, protocols, and applications. In Communications and Networking in China, 2008. ChinaCom 2008. Third International Conference. IEEE, 2008.

[7] Mahdi Asadpour, Simon Egli, Karin Anna Hummel, and Domenico Giustiniano. Routing in a fleet of micro aerial vehicles: First experimental insights. In conjunction with the 15th ACM International Symposium on Mobile Ad Hoc Networking and Computing (ACM MobiHoc 2014), Philadelphia, PA, USA., 11 August 2014.

[8] Jürgen Scherer, Saeed Yahyanejad, Samira Hayat, Evsen Yanmaz, Torsten Andre, Asif Khan, Vladimir Vukadinovic, Christian Bettstetter, Hermann Hellwagner, and Bernhard Rinner. An autonomous multi-uav system for search and rescue. Technical report, Institute of Networked and Embedded Systems and Institute of Information Technology (ITEC) Alpen-Adria Universität Klagenfurt, Austria Lakeside Labs, Klagenfurt, Austria, 2015.

[9] Kurt Konoligeand Dieter Fox, Benson Limketkai, Jonathan Ko, and Benjamin Stewart. Map merging for distributed robot navigation. In Intelligent Robots and Systems. IEEE, 2003.

[10] A. Cunningham, M. Paluri, and F. Dellaert. Ddf-sam: Fully distributed slam using constrained factor graphs. In Intelligent Robots and Systems (IROS), 2010 IEEE/RSJ International Conference. IEEE, 2010.

[11] G. Klein and D. Murray. Parallel tracking and mapping for small ar workspaces. In Mixed and Augmented Reality, 2007. ISMAR 2007. 6th IEEE and ACM International Symposium. IEEE, 2007.

[12] Duy-Nguyen Ta, Kyel Ok, and Frank Dellaert. Monocular parallel tracking and mapping with odometry fusion for mav navigation in feature-lacking environments. Institute for Robotics and Intelligent Machines (IRIM), 2013.

[13] C. Brand, M.J. Schuster, H. Hirschmuller, and M. Suppa. Stereo-vision based obstacle mapping for indoor/outdoor slam. IROS, 2014.

[14] Wang Qing, Giustiniano Domenico, and Puccinelli Daniele. Openvlc: Software-defined visible light embedded networks. In The 1st ACM Workshop on Visible Light Communication Systems, in conjunction with the 20th Annual International Conference on Mobile Computing and Networking (ACM MobiCom 2014), Maui, Hawaii, USA., 2014. 
[15] Qing Wang and Domenico Giustiniano. Communication networks of visible light emitting diodes with intra-frame bidirectional transmission. In The 10th ACM International Conference on emerging Networking EXperiments and Technologies (ACM CoNEXT 2014), 2-5 December 2014, Sydney, Australia, 2014.

[16] T. Zahariadis and S. Voliotis. Open issues in wireless visual sensor networking. In Systems, Signals and Image Processing, 2007 and 6th EURASIP Conference focused on Speech and Image Processing, Multimedia Communications and Services. 14th International Workshop on, 2007.

[17] Ilker Bekmezci, Ozgur Koray Sahingoz, and Samil Temel. Flying ad-hoc networks (fanets): A survey. Ad Hoc Networks, 2013.

[18] Sonia Waharte, Niki Trigoni, and Simon J. Julier. Coordinated search with a swarm of uavs. Sensor, Mesh and Ad Hoc Communications and Networks Workshops, 2009. SECON Workshops '09. 6th Annual IEEE Communications Society Conference on, 2009.

[19] Stephen Cameron and Niki Trigoni. Collaborative sensing by unmanned aerial vehicles. In 3rd International Workshop on Agent Technology for Sensor Networks (ATSN 09), 2009.

[20] T. Imboden, K. akkaya, and Z. Moore. Performance evaluation of wireless mesh networks using ieee 802.11s and ieee 802.11n. Communications (ICC), IEEE, 2012.

[21] J.C.-P. Wang, B. Hagelstein, and M. Abolhasan. Experimental evaluation of ieee 802.11s path selection protocols in a mesh testbed. In Signal Processing and Communication Systems (ICSPCS). IEEE, 2010. 